\title{
Energy-efficient internetworking with DTN
}

\author{
Dimitris Vardalis ${ }^{1}$ and Vassilis Tsaoussidis ${ }^{2}$ \\ ${ }^{1}$ Space Internetworks Ltd, 12 Rethymnou, 10682 Athens, Greece \\ ${ }^{2}$ Space Internetworking Center, Dept Of Electrical and Computer Engineering, \\ Demokritos University of Thrace, Xanthi 67100, Greece
}

\begin{abstract}
We claim that Delay-Tolerant Networking has the potential to form an internetworking overlay that shapes traffic in a manner that exploits the capacity of last hop wireless channels and allows for energy-efficient internetworking. We demonstrate DTN potential for energy-efficient internetworking through an overlay-architecture and a tool we have developed that captures the required state transitions of the mobile device WNIC. We show experimentally that the DTN overlay can re-shape traffic in a manner that allows the receiver to exploit the energy-throughput tradeoff better: it may condense sporadic packets into a burst and, in return, prolong sleep and power-off duration without risk to miss incoming packets and without degradation in throughput.
\end{abstract}

Keywords: DTN, energy efficiency, internetworking

\section{Introduction}

Mobile devices became a useful every-day tool for communication, storage and entertainment. They are equipped with increasingly more powerful CPUs, larger memory capacities, faster wireless network adapters, and a set of applications that require internetworking capabilities. However, advances in battery technology have not been able to match the increased energy demand.

So far, the main focus of attention was the energy-efficient administration of device components, the selection of routing with energy capacity to route packets, or the capacity of battery itself. The (inter-) network per se has never attested its vital role in energy efficiency. The Internet, due to its probabilistic nature constitutes a major reason for the lack of enddevice energy control. Its scheduling uncertainty forces a receiver on continuously waiting for random contacts and, inevitably, enforces an energy-wasting policy; as a result, the device cannot incorporate energy-efficient modes of operation, such as sleep, into its transition state diagram. Therefore, a main reason for the inability to optimize end-device operations is the inherent inability of the internetwork to deliver data tactically, due to its store-and-forward and statistical multiplexing characteristics. Practically, what imposes the limitation to schedule transmission with accurate precision is the variation of delay, along with the potential lack of sufficient resources to store packets in transit, albeit having such a capacity could have, instead, permitted the network to shape traffic and corroborate a scheduling policy among the devices and the network.

In this work, we attempt to demonstrate the potential of Delay Tolerant Networking (DTN) ([1], [2]) to shape internetwork traffic in a manner that allows mobile devices to balance their energy expenditure with minimal cost on throughput. In this context, we design and deploy an internetworking overlay that exploits two major DTN properties: (i) to store packets for as long as it is necessary, regardless of disruptions in communications; and (ii) to enhance the edge nodes with functionality to wait for sufficient amount of packets to arrive, prior to transmitting to the end node. We then develop scenarios and weigh our expectations using a 
broad set of experiments that reflect potential variations in traffic characteristics, error probabilities and disruptions.

In order to evaluate the proposed scheme we use the ns-2 network simulator. Since there is no established and reliable DTN implementation for ns-2 we opted for emulating the behavior of the bundle protocol by introducing a proxy application at the base station. The energy expenditure model of ns-2 was also not sufficient for this work, since the whole state transition diagram for the WNIC had to be recorded. Hence, energy expenditure calculations involved hacking into the physical layer of the wireless node and adding tracing for the state transitions. Our results show that (i) energy expenditure can be reduced drastically and (ii) clearly, DTN appears as an appropriate tool to construct such overlay: it allows for permanent storage and, in addition, it does not require full, but instead, partial deployment on top of IP; in fact, even a minimal deployment of DTN nodes can satisfy the architectural requirements of energy-saving overlay as soon as the edge nodes deploy such service.

The rest of the paper is organized as follows: In section 2 we discuss related work, focusing on more recent approaches to save energy on mobile devices. In section 3 we discuss our proposal and in section 4 we detail our experimental tools and methodology, including parameters for evaluation, metrics and scenarios developed. In section 5 we discuss the results and, finally, in section 6 we summarize our conclusions.

\section{Related Work}

The research literature on energy efficiency for mobile devices includes two broad categories that correspond to the device optimization and communication optimization. In [3], the authors identify as the main sources of energy expenditure the various subsystems of a handheld device, such as: the processor, the memory, the display, the audio system and the wireless networking. For network intensive applications, the power consumption of the Wireless Network Interface Controller (WNIC) can reach up to $60 \%$ of the total power necessary for the operation of the mobile device [4]. In [5] Jones et al. provide a comprehensive survey on the design principles of efficient network protocols. The authors focus on the specific mechanisms that can be employed in each of the layers in the network protocol stack (i.e. Physical, Data Link, Network, Transport, OS/Middleware and Application).

In [6], Adams et al. propose a buffering technique that exploits the inherent power-saving mode of 802.11. Data destined to a certain mobile node is hidden and thus, not made available to it for multiple beacon intervals. Mamatas and Tsaoussidis in [7] monitor the state transition of the WNIC and introduce two metrics for energy potential and unexploited available resource, to capture the energy performance potential of various protocols. The authors in [8] present a theoretical and empirical study of the impact of packet transmission interval and burst length on observed network performance characteristics, with or without use of a proxy service. They propose an adaptive, energy-saving streaming mechanism that adjusts the burst length. By the same token, however now focusing generally on TCP traffic, Anastasi et al. [9] introduce at the base station a proxy service that employs regular TCP on the wired network side and the novel Power-Saving Transmission Protocol on the wireless side. Authors in [10] expand on the proxy idea by introducing a scheduler service at the base station and a proxy at the mobile terminal. The scheduler incorporates a novel algorithm, called priority-based bulk scheduling (PBS). In [11] the proxy idea is again implemented at the base station introducing the novel RT_PS protocol for the communication between the base station and the mobile node. The RT_PS protocol includes idle intervals information so that the client can put the WNIC into sleep mode when applicable. By the same token, Batsiolas and Nikolaidis [12] 
propose a regulation of ACKs for TCP in a manner that allows the sender to predict with some accuracy when ACKs are expected; and meanwhile the sender enters a sleep mode.

\section{Energy-efficient internetworking overlay}

We depart from the mobile device modus operandi: at any given moment, a WNIC can be in one of five operation states, each with different power requirements: transmit, receive, idle, sleep (or doze) and power-off. The transmit, receive and idle state of the WNIC constitute the active states, whereas the sleep and power-off constitute the inactive states. The power requirements of active states are comparable with each other [3]. In the transmit state maximum power is consumed, while in the receive state slightly more power is required than in the idle state. In the sleep state the energy expenditure is an order of magnitude less than that of the idle state, and finally, in the power-off state no power is required. The IEEE 802.11 [13] protocol provides an inherent mechanism for allowing client devices to manage the power requirements of their WNICs. The mechanism relies on the central role played by the Access Point when the network is in the PCF (Point Coordination Function) operation mode (in this paper we do not consider the ad-hoc or Distributed Coordination Function of 802.11).

It becomes apparent that the challenge in energy-saving strategies now translates into how to maximize the duration that the WNIC spends in either the sleep or the power-off state. It is obvious, however, that the AP has a limited buffer and therefore, the scheduling precision and flow control of the AP determine the efficiency of each strategy. Otherwise, the risk of entering a sleep mode may lead to costly retransmissions and throughput degradation due to buffer overflow or receiver inactivity. Data buffering essentially shapes traffic in bursts so that periods of inactivity are prolonged, allowing the WNIC to stay in a low-power state longer, while at the same time minimizing the performance trade-off with throughput. Hence, it makes sense to design an overlay that buffers at least a Delay X Bandwidth product (DxB) between the edge node and the receiver (prior to transmitting) and distributes data storage among nodes in a manner that packet dropping is avoided. This sort of sophistication has not been developed or analyzed in our present work. We construct a rather demonstrative overlay where buffers have enough space; the disruptions never go beyond the storage capacity, so the possibility to cause buffer overflow is cancelled, in practice. In this context, we evaluate only the ability of the overlay to shape traffic in a manner that optimizes the transitions of the mobile device; and we evaluate the characteristics of the tradeoff with throughput.

The pseudo-code of the algorithm at a DTN node of the overlay is as follows:

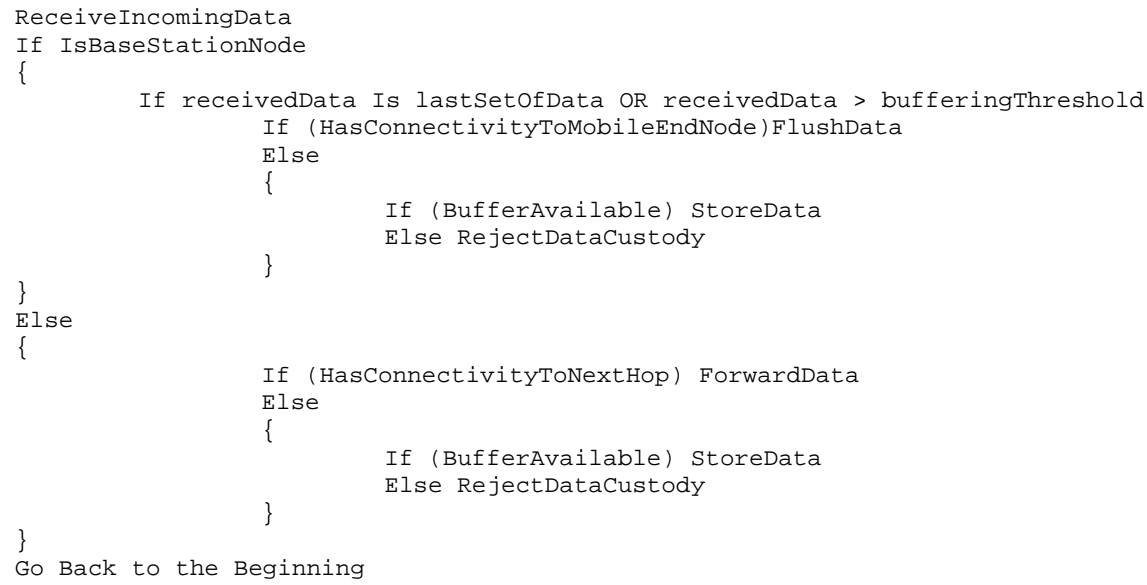


What we also consider as novel in our proposal is the ability of the edge node (i.e. the last DTN node) to make a decision locally, with better precision than the sender of a typical endto-end application. The architecture has the potential to exploit further the custody property of the DTN protocol, which leads to an automated balancing of storage as a result of the operation to shift data towards the destination only when the accepting nodes do have storage availability.

\section{Experimental methodology}

We emulated the behavior of the bundle protocol by introducing a proxy application at the base station. Measuring the energy expenditure was made possible by directly monitoring the physical layer of the receiver and making log entries for each state change of the WNIC. The proxy application is associated with an incoming TCP connection originating from some source on the wired part of the network and an outgoing TCP connection that ends at the wireless receiver. The proxy application buffers data coming in from its receiving end and flushes it out to its sending end as soon as the desired amount of data is collected. This setup emulates the functionality of a DTN overlay on an IP network, when using TCP as the convergence layer.

The proxy application was implemented as a subclass of the Application class in ns-2, adding the required extra functionality. Additional functionality implemented within the new class includes: an agent that receives data from the wired source, an option for the buffer size and an option for the total size of the transfer. The proxy needs to be aware of the total transfer size so that, when the specified amount of data is received, the buffer is immediately flushed without waiting for the buffering threshold to be reached.

In order to facilitate the energy expenditure calculations we modified the physical layer of the wireless node. Code that traces the duration the WNIC spends in each of the three states (i.e. send, receive and idle) was added. By having an accurate picture of state transitions we are able to carry out post simulation calculations and find the potential energy savings if there was in place a mechanism that would switch the WNIC into the sleep state during idle intervals. In this paper we aim at emphasizing the energy saving potential that a DTN-based protocol can have for mobile devices. Addressing various optimization aspects, including the inherent load balancing capability of DTN custody transfer, is part of our future work plans.

\subsection{Energy expenditure calculations and comparison.}

During the post-simulation analysis a multi-purpose script identifies the energy-related log entries in the ns-2 trace file and calculates the energy expenditure based on certain input parameters. The script takes the following input parameters: transmit power (txpower), receive power (rxPower), idle power (idlePower), sleep power (sleepPower), transition power (transPower) and transition time (transTime). For the transmit and receive intervals the energy expenditure is calculated as the duration of the interval multiplied by the transmit and receive power respectively. Energy conservation can be achieved by exploiting the idle intervals and switching into the sleep state. The transition is considered feasible only if the time it takes to fall into the sleep state and then back to the idle state (i.e. transTime) is less than the duration of the idle interval itself. The transition is considered meaningful if the energy required for switching back and forth to the sleep state, plus the energy spent during the sleep state is less than the energy expenditure if the WNIC had remained in an idle state for the whole interval duration. If idleDuration is the duration of the idle interval, the decision is based on the following formula: 


$$
2 \text { * transPower * transTime + (idleDuration - } 2 \text { * transTime }) \text { * sleepPower < }
$$

idleDuration * idlePower

It becomes obvious that the longer the duration of the idle intervals the greatest the opportunity for conserving energy. The power figures for the various WNIC states are set as follows [14]: txPower $=1.400$ Watts, $r x$ Power $=0.950$ Watts, idlePower $=0.805$ Watts and sleepPower $=0.060$ Watts. As reported in [15] transition time from sleep to idle and vice versa, is in the order of tens of milliseconds. However, when switching back to the active state, the controller needs to wait for the next beacon in order to start receiving the buffered data. If we assume that it takes $50 \mathrm{~ms}$ to switch states and another $100 \mathrm{~ms}$ for the next beacon signal to be broadcast (a rather pessimistic estimation since $100 \mathrm{~ms}$ is the default beacon interval for 802.11) the total for the transition amounts to $200 \mathrm{~ms}$. Therefore, we set the transition delay at $100 \mathrm{~ms}$ for both state transitions. Finally, based on measurements in [16] we can safely consider the energy expenditure in the transitive state to be the same as that of the idle state (i.e. transpower $=0.805$ ).

\subsection{Model description}

Figure 1 depicts the network topology that was implemented in order to evaluate the energy conservation that could be achieved by employing a DTN-like protocol in wired-cum-wireless scenarios. Network nodes are named as N1 - N6. N4 is the base station node that is connected to both the wired and the wireless networks and node N6 is the wireless receiver. Links L13, L23, L34 and L45 are wired, while WL is the wireless link between the base station and the receiver.

N6

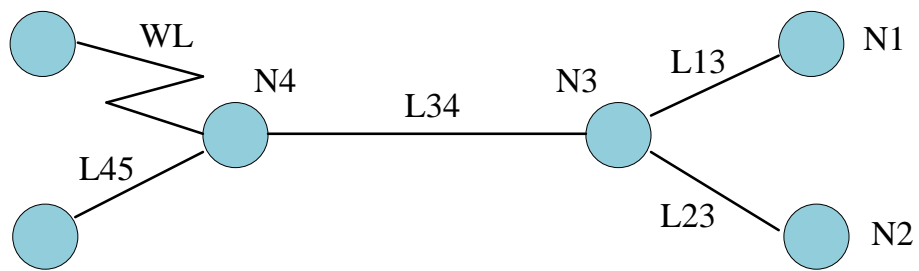

Figure 1: Network Topology.

The data transfer studied in this experiment follows the $\mathrm{N} 1 \rightarrow \mathrm{N} 3 \rightarrow \mathrm{N} 4 \rightarrow \mathrm{N} 6$ route, while the competing flow follows the $\mathrm{N} 2 \rightarrow \mathrm{N} 3 \rightarrow \mathrm{N} 4 \rightarrow \mathrm{N} 5$ route. The bandwidth of the backbone link (L34) is changed so that the desired congestion is introduced into the network. The rest of the links are kept at the same delay and bandwidth values for all the experiments.

The bandwidth and delay values for all the links are as follows: L13 - 2Mb, 100ms, L23 $3 \mathrm{Mb}, 100 \mathrm{~ms}$, L34 - 300ms delay and varying bandwidth depending on the desired network characteristic, L45 - 3Mb, 100ms, WL - 802.11 with a data rate of $11 \mathrm{Mb}$ and a basic rate of $1 \mathrm{Mb}$. Stochastic Fair Queuing is applied on all queues, the file transfer size is $10 \mathrm{MB}$ and, when present, the competing flow sends a 1000 byte packet every $3 \mathrm{~ms}$. TCP packet size is 1460 and maximum window size 100 packets.

The resulting energy expenditure values are in Joule. In the End-to-end (E2E) scenarios there is an end-to-end connection between N1 and N6, whereas in the Split scenarios there is a splitting application on the base station acting as a transport layer mediator. In order to measure the delay of the wireless connection between the base station and the mobile receive we employed a ping agent. The delay was found to be around $2 \mathrm{~ms}$. The DxB is thus: $0.002 \mathrm{x}$ $11000000 / 8=2.750 \mathrm{~KB}$. Except otherwise noted, the splitting application buffers $30 \mathrm{~KB}$ (around $10 \mathrm{x}$ the $\mathrm{DxB}$ ) before forwarding them to the mobile device. In case the wireless 
connection is the bottleneck and data piles up at the base station we make the assumption that there is no limit in the available memory used for buffering data and, therefore, no data is dropped.

Finally, experiments are carried out both for an FTP connection transferring $10 \mathrm{MB}$ of data as well as CBR traffic that flows for a specified amount of time. In certain experiments we are introducing a packet error rate on the wireless LAN. The error follows a uniform distribution and is applied on the outgoing interface of both the base station interface as well as the WNIC of the mobile node.

\section{Results}

In this section we present the experimental results, organized in five subsections of experimental setups. Due to limited space we only present a subset of the available results. A full version of the results can be found in [17]. The setups presented in this paper are as follows: FTP and varying wired bandwidth (with and without a competing flow), FTP and varying wireless error (with and without a competing flow), FTP and varying buffering threshold (with and without a competing flow), CBR and varying buffering threshold.

\subsection{Varying Bandwidth FTP Transfer}

In this set of experiments the backbone link is assigned a constant delay of 300ms, while the bandwidth varies from $0.5 \mathrm{Mb}$ to $2.5 \mathrm{Mb}$ in steps of $0.5 \mathrm{Mb}$. The total transfer duration (Table 1) is virtually the same for both the E2E and the Split case, and it is greatly affected by the bandwidth of the backbone link because of network congestion. Since the upstream link (L13) has a $2 \mathrm{Mb}$ bandwidth, setting the backbone bandwidth to values greater than $2 \mathrm{Mb}$ does not affect simulation results (L13 becomes the bottleneck of the connection and the backbone is uderutilized). Because of this, the transfer duration is equal for bandwidth values of $2 \mathrm{Mb}$ and 2.5Mb.

In terms of the energy efficiency, it is obvious from the chart of Figure 2 that smaller bandwidth values for the bottleneck link lead to greater energy gains of the Split approach. In the extreme setting of the $0.5 \mathrm{Mb}$ bandwidth, the energy conservation achieved in the Split case is approximately $44 \%$ of the energy expenditure in the E2E case.

As the network congestion is eased, due to the increase of the bottleneck link capacity, the energy conservation becomes less dramatic and it reaches the 5 Joule value. When severe network congestion is present on the network TCP introduces considerable overhead in its effort to achieve reliable transmission. In the E2E case, the mobile node at the receiving end is involved in this extra effort and, consequently, does not frequently have the opportunity to switch to sleep mode. Conversely, in the Split case the base station absorbs the effects of the network congestion (lost packets that lead to out-of-order data reception) and only transmits to the mobile host when consequent, buffer-sized chunks of data are available. In DTN terms, the base station will be receiving the incoming bundle without engaging the receiving node in the process. Upon successful bundle reception forwarding to the mobile node would start. In the meantime, the WNIC of the mobile host can switch to sleep mode and save significant amounts of energy. The higher the congestion level the greater the energy gains achieved by the Split approach. In the case where Power Saving Mode (PSM) is not employed, the energy expenditure is again related to the transfer duration. In the $0.5 \mathrm{Mb}$ bandwidth setting the No PSM energy expenditure is twice as much as that of the Split case, dropping to about $50 \%$ more than the Split case in the $2.5 \mathrm{Mb}$ setting. 


\begin{tabular}{|c|c|c|}
\hline $\begin{array}{c}\text { L34 bandwidth } \\
(\mathrm{Mb})\end{array}$ & $\begin{array}{c}\text { Duration E2E } \\
(\mathrm{sec})\end{array}$ & $\begin{array}{c}\text { Duration Split } \\
(\mathrm{sec})\end{array}$ \\
\hline 0.5 & 216.85 & 216.05 \\
\hline 1 & 145.59 & 144.88 \\
\hline 1.5 & 111.65 & 111.17 \\
\hline 2 & 90.48 & 90.18 \\
\hline 2.5 & 90.34 & 90.04 \\
\hline
\end{tabular}

Table 1: FTP, Varying Bandwidth, No Competing Flow Transfer Duration.

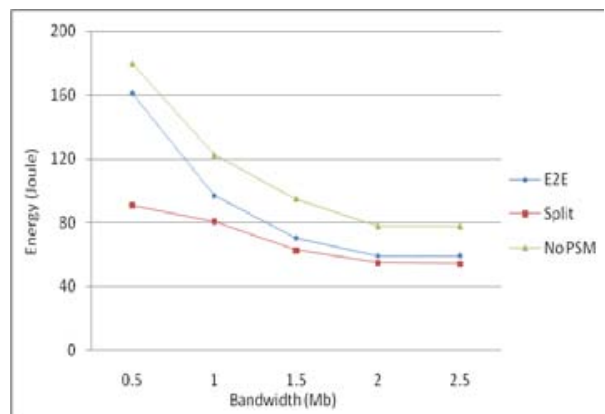

Figure 2: FTP, Varying Bandwidth, No Competing Flow Energy Expenditure.

In the previous scenario we added cross traffic and rerun the experiments. In this set of experiments the maximum bandwidth value for the backbone link was increased to 3.5, since beyond that capacity the measurements remain unchanged.

\begin{tabular}{|l|c|c|}
\hline $\begin{array}{l}\text { L34 bandwidth } \\
(\mathrm{Mb})\end{array}$ & $\begin{array}{l}\text { Duration E2E } \\
(\mathrm{sec})\end{array}$ & $\begin{array}{l}\text { Duration Split } \\
(\mathrm{sec})\end{array}$ \\
\hline 0.5 & 364.61 & 357.91 \\
\hline 1 & 225.85 & 194.47 \\
\hline 1.5 & 159.32 & 158.63 \\
\hline 2 & 125.39 & 124.53 \\
\hline 2.5 & 107.72 & 107.21 \\
\hline 3 & 90.04 & 89.73 \\
\hline 3.5 & 90.74 & 90.25 \\
\hline
\end{tabular}

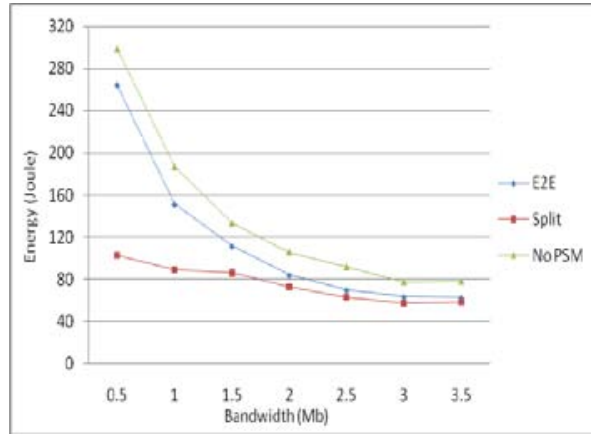

Table 2: FTP, Varying Bandwidth, Competing Flow Transfer Duration.

Figure 3: FTP, Varying Bandwidth, Competing Flow Transfer Duration.

As expected, the transfer duration, shown in Table 2, is significantly longer for both the E2E and the Split approaches when a competing flow is present. Nevertheless, unlike the previous scenario, small bandwidth values (i.e. 0.5 and $1 \mathrm{Mb}$ ) result to a slightly shorter transfer duration for the Split case.

Regarding the energy consumption, it can be observed in Figure 3 that despite the significantly longer time it takes for the transfer to complete for the $0.5 \mathrm{Mb}$ setting (around $60 \%$ more in both cases), in the E2E approach the additional energy requirement is $62 \%$, while in the Split approach the additional energy requirement is only around $10 \%$. As the bandwidth increases and the network is relieved from congestion, the energy conserved by the Split approach is limited to around 5 Joule (a value that was observed in almost all congestionfree experiments).

Figure 4 depicts the state transitions for the first 14 seconds of the file transfer in both the E2E and the Split cases when the backbone bandwidth is $1 \mathrm{Mb}$. It is obvious from the chart that in the E2E case, the WNIC needs to switch to active more frequently than in the Split case. For example, between 2 and 6 seconds, in the E2E case the WNIC needs to switch to active 4 times, while in the Split case it remains in the sleep state for the whole duration. Also, 
even when a switch to the active state is necessary the time needed to remain in this state is shorter for the Split than for the E2E scenario (i.e. at 6, 7, 11 seconds).

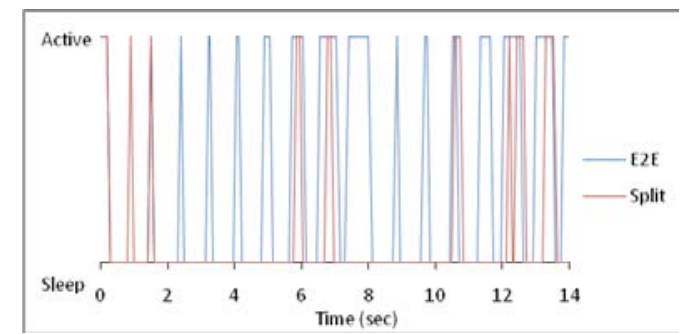

Figure 4: FTP, 1Mb Bandwidth, Competing Flow State Transitions.

\subsection{Varying Wireless Error FTP Transfer}

The purpose of this scenario is to reveal the effect of packet error on the wireless part of the network both on the transfer duration as well as the energy efficiency of the E2E and the Split approaches. The backbone link was assigned a 300ms delay and a $1 \mathrm{Mb}$ bandwidth. The delay was selected so that it is considerably longer than the delays of the rest of the links in the topology and the bandwidth was selected so that the backbone is also the bottleneck link of the transfer route (i.e. network congestion is present).

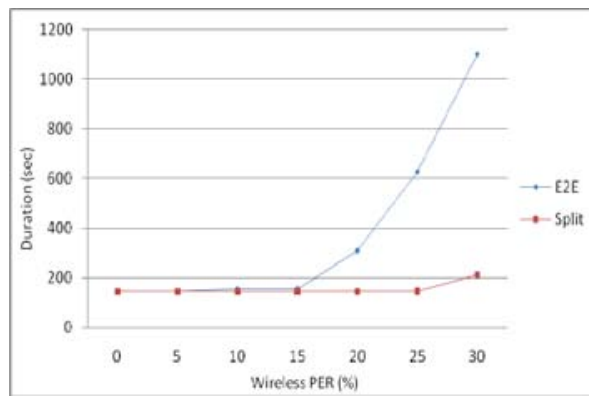

Figure 5: FTP, Varying Wireless PER, No Competing Flow Duration.

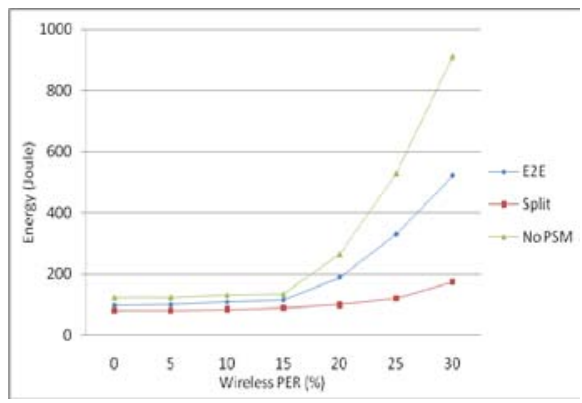

Figure 6: FTP, Varying Wireless PER, No Competing Flow Energy Consumption.

At first glance, it may be striking that as the wireless packet error rate (PER) increases the duration of the E2E transfer increases as well (Figure 5), reaching in the 25\% case more than three times the duration of the $0 \%$ case, whereas the duration of the Split case transfer remains almost the same across all runs. Part of the explanation to this, seemingly bizarre, result can be found in the Indirect-TCP solution proposed in [18]. In the E2E scenario, it takes very long for TCP to recover from packets lost due to the wireless error, because of the long RTT of the connection (around $800 \mathrm{~ms}$ ). On the contrary, the RTT of the wireless link is measured to be around 6-7 ms, and, therefore, a TCP connection between the base station and the mobile host can be very swift in recovering the lost packets. Additionally, since the RTT of the wired part of the route is very long, when compared to that of the wireless part, the time it takes for the base station to buffer the required data is enough for the TCP of the last hop to recover from any packet losses. This observation is not true only for the 30\% PER case, in which it is evident that the last hop TCP cannot serve the incoming data, delaying the overall transfer time. In such a case, data could be dropped at the base station if the buffer capacity is depleted. We plan to include monitoring of packets dropped due to limited buffer capacity in 
future experiments. The performance gains of a solution along the lines of Indirect-TCP that isolates the wireless error in the short-delay part of the transfer route come naturally as additional benefit when using DTN.

The energy consumption chart in Figure 6 shows that up to an error rate of 10\% the energy savings achieved by the Split approach are approximately $16 \%-20 \%$. As the error rate increases so does the conserved energy, reaching as much as $66 \%$ for an error rate of $30 \%$. At this error rate the energy required for the completion of the data transfer in the E2E case is 520.78 Joule, while in the Split case the required energy is 174.09 Joule. The energy expenditure in the No PSM case for the $30 \%$ error rate is as much as 910.49 Joule.

The state transitions for an indicative 15 second interval when the wireless PER is $20 \%$ are plotted in the chart of Figure 7. It can be observed that, for the whole duration covered in the chart, the WNIC switches to the active state 14 times in the E2E case, while it only switches 6 times in the Split case. The state transition frequency for the two cases is similar during the rest of the data transfer, resulting in the significant energy expenditure difference observed in Figure 6 for a $20 \%$ PER.

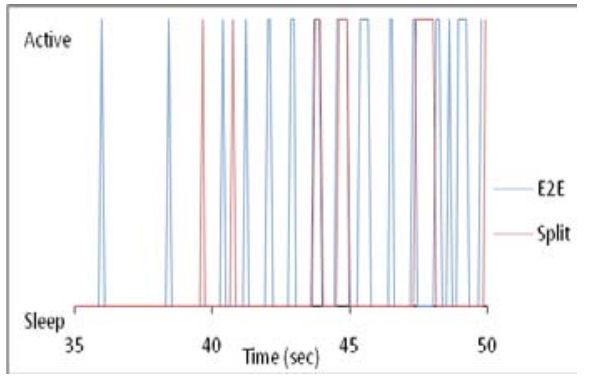

Figure 7: FTP, 1Mb Bandwidth, 20\% Wireless PER, No Competing Flow State Transitions.

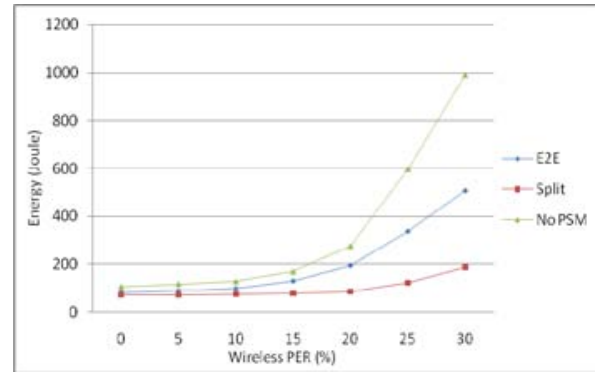

Figure 8: FTP, Varying Wireless PER, Competing Flow, Energy Consumption.

The experiments in the previous section were repeated with a competing flow present on the network. Because of the huge transfer times that would otherwise be necessary, the bandwidth of the backbone link was set to $2 \mathrm{Mb}$ (as opposed to the $1 \mathrm{Mb}$ value that was used in the previous section). Even though we cannot directly compare the resulting figures for the cases with or without a competing flow, we can still draw meaningful conclusions for the relative performance of the E2E vs. the Split case. The most significant observation in this scenario as depicted in Figure 8 is that when a competing flow is present, the energy expenditure of the E2E approach exhibits higher increase rates for smaller error rates. For higher error rates both the competing and the non-competing flow cases exhibit similar behavior.

\subsection{Varying Buffer Threshold FTP Transfer}

In this set of experimental runs the delay is set to $300 \mathrm{~ms}$, the bandwidth of the backbone link is set to $1 \mathrm{Mb}$ (rendering it as the bottleneck link of the transfer route) and the buffering size varies from 0 up to $200 \mathrm{~KB}$. For the 0 buffering case we used the E2E approach, while for the rest of the runs we used the Split approach with the corresponding buffer size. The transfer duration is, reasonably, identical for all buffer sizes. The energy expenditure, however, drops significantly as the buffer size increases (Figure 9). Since the wireless link of the transfer route is consistently underutilized, buffering more data allows the WNIC of the receiver to switch to 
a sleep state for longer periods, while at the same time not getting penalized for waiting. Increasing the buffer size beyond a certain value could cause congestion problems on the wireless LAN and/or prolong the transfer duration. Such problems could become more severe in case multiple mobile clients are present and download data using the Split approach. With the increase in the buffering capacity the traffic on the wireless LAN becomes more bursty and consequently more prone to congestion. The size of the buffer, in case DTN is employed, can be thought of as the size of the bundle size that the base station would forward to the last hop of the transfer connection.

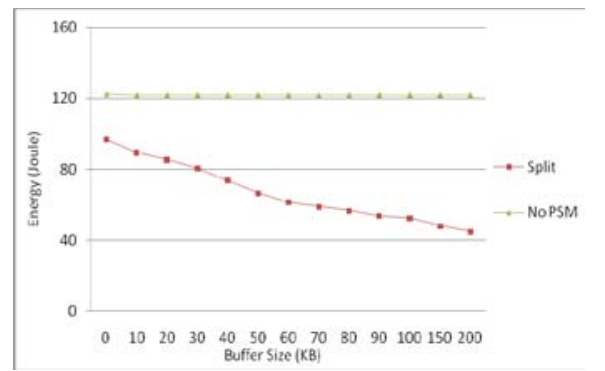

Figure 9: FTP, Varying Buffering Threshold, FTP, No Competing Flow, Energy Consumption.

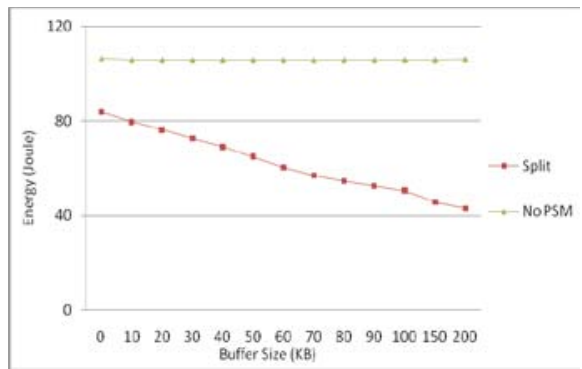

Figure 10: FTP, Varying Buffering Threshold, Competing Flow, Energy Consumption.

The experiments of the previous scenario were repeated with the introduction of cross traffic caused by a competing flow. In order to compensate for the higher network traffic the bandwidth of the backbone link was increased to $2 \mathrm{Mb}$. In both sets of experiments the transfer duration remains constant for all runs. With respect to the energy expenditure, shown in Figure 10 it is observed that while for small buffer sizes the amount of energy required by the no competing flow scenario is significantly higher, as the buffer size increases the two values converge to a common Joule value in the low 40s. This shows that increasing the buffer size can alleviate differences in the energy expenditure caused by varying congestion conditions in the wired part of the network.

\subsection{Varying Buffer Threshold CBR Transfer}

In this last set of experiments a CBR traffic flow of 10MB is considered in a network with no contending traffic. As in most of the previous scenarios the delay of the backbone link was set to $300 \mathrm{~ms}$ and the bandwidth to $2 \mathrm{Mb}$. The transfer duration does not convey any important information as it merely refers to the time the last packet is received by the mobile node and it depends solely on the end-to-end network delay.

This scenario can be mostly considered as a placeholder for future work, since the side effects of delaying CBR traffic are not taken into account. When measuring the service quality of a file transfer the time for completion can be an adequate metric. The sooner the file transfer finishes the better it is for the end user. In all the experiments, so far, the transfer duration was not affected by the buffering mechanism, rendering the mechanism acceptable from a user standpoint. However, CBR traffic is, in most cases, related to multimedia streaming. Extra delays introduced by the buffering mechanism can lead to unacceptable service quality, a factor which is not captured by our current experimental setup. Nevertheless, 
the experiments in this scenario reveal the potential a DTN-based Split approach can have for reducing the energy consumption for CBR traffic.

It becomes apparent from the chart in Figure 11 that even for a buffer size of 20KB, the energy saving potential is $33 \%$ compared to the case where no power saving is used (No $\mathrm{PSM})$. As the buffering size increases the conserved energy increases as well, reaching almost $85 \%$ of the energy spent in the No PSM case. Although large buffering in multimedia streams can cause long delays and, thus, unacceptable user experience, if used with caution it can deliver significant energy conservation with little or no quality degradation.

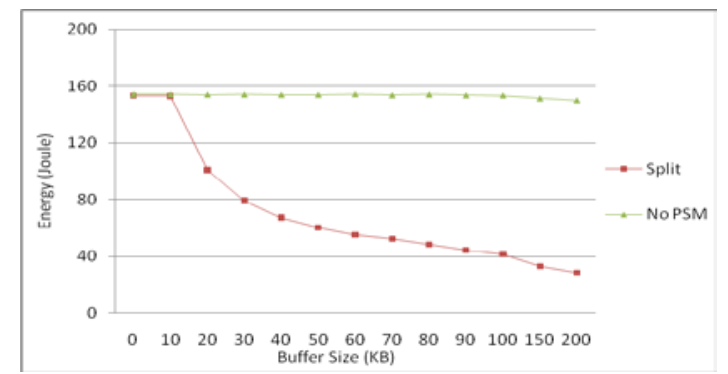

Figure 11: CBR, Varying Buffering Threshold Energy Consumption.

\section{Conclusions \& Future Work}

We evaluated the potential of DTN to shape Internet traffic in a manner that allows mobile devices to utilize energy-conserving modes of operation, without risk to lose packets or degrade their throughput. Our results are based on a post-simulation analysis of the recorded transition state diagram of the mobile device, as this was determined by the communication between the end-device and the edge network node. The most conclusive results of our experiments capture the huge gain in useless transitions and uncover the energy potential of DTN properties.

We briefly discussed the property of DTN to balance network traffic as well. This is not peripheral to energy-saving strategies: Load balancing within the network, unlike typical endto-end approaches, cancels the risk of packet dropping but also forwards information towards the destination as soon as possible. Application data, instead of waiting at the source, it is pushed at some network nodes closer to the destination. This latter becomes particularly important in environments, such as space for example, where a contact graph is predetermined and the penalty for losing a contact opportunity may delay communication significantly. Hence, load balancing strictly associates with less retransmissions and shortened communication time: two tactics with energy-conserving consequences. We plan to focus on this dimension of energy-saving strategies by extending our present evaluation framework with load balancing algorithms.

Finally, taming uncertainty allows for more sophisticated signaling techniques that deploy a hybrid Time Division and Statistical Multiplexing strategy. This sort of sophistication is under progress.

\section{References}

[1] V. Cerf, S. Burleigh, A. Hooke, L. Torgerson, R. Durst, K. Scott, K. Fall, and H. Weiss, "Delay-Tolerant Networking Architecture”, RFC 4838, IETF, 2007.

[2] K. Scott and S. Burleigh, “Bundle Protocol Specification”, RFC 5050, IETF, 2007. 
[3] M. Viredaz, L. Brakmo, and W. Hamburgen, "Energy Management on Handheld Devices”, ACM Queue Magazine - Power Management, Volume 1 Issue 7, 2003.

[4] A. Acquaviva, T. Simunic, V. Deolalikar, and S. Roy, "Remote power control of wireless network interfaces”, Proceedings of International Workshop on Power and Timing Modeling, Optimization and Simulation, 2003.

[5] C. Jones, K. Sivalingam, P. Agrawal, J. Chen, “A Survey of Energy Efficient Network Protocols for Wireless Networks”, ACM Journal on Wireless Networks, Volume 7, Issue 4, 2001.

[6] J. Adams and G.M. Muntean, “Adaptive-Buffer Power Save Mechanism for Mobile Multimedia Streaming,” Proceedings of IEEE International Conference on Communications '07, 2007.

[7] L. Mamatas, V. Tsaoussidis, "Exploiting Energy-Saving Potential in Heterogeneous Networks", International Journal of Parallel, Emergent and Distributed Systems (IJPEDS), Taylor \& Francis, volume 23, issue 4, 2008.

[8] J. Korhonen and Y. Wang, "Power-efficient streaming for mobile terminals”, Proceedings of ACM International Workshope on Network and Operating Systems Support for Digital Audio and Video, 2005.

[9] G. Anastasi, M. Conti, and W. Lapenna, "A Power-Saving Network Architecture for Accessing the Internet from Mobile Computers: Design, Implementation and Measurements ”, The Computer Journal, Volume 46, Issue 5, 2003.

[10] H. Zhu and G Cao, "A Power-Aware and QoS-Aware Service Model on Wireless Networks ”, Proceedings of IEEE INFOCOM '04, 2004.

[11] G. Anastasi, M. Conti, E. Gregori, A. Passarella, and L. Pelusi, "A Power-Aware Multimedia Streaming Protocol for Mobile Users", Proceedings of the IEEE International Conference on Pervasive Services '05, 2005.

[12] I. Batsiolas and I. Nikolaidis, "Selective Idling: Experiments in Transport Layer Energy Conservation”, The Journal of Supercomputing, Volume 20, Number 2, 2001.

[13] IEEE Standard for Information technology - Telecommunications and information exchange between systems - Local and metropolitan area networks - Specific requirements , Part 11: Wireless LAN Medium Access Control (MAC) and Physical Layer (PHY) Specifications , 2007.

[14] Eugene Shih, Paramvir Bahl, and Michael J. Sinclair, "Wake on Wireless: An Event DrivenEnergy Saving Strategy for Battery Operated Devices", Proceedings of the ACM International Conference on Mobile Computing and Networking, 2002.

[15] T. Simunic and S. Boyd, "Managing power consumption in networks on chips," ACM

DATE, 2002.

[16] Yu Xiao, Petri Savolainen, Arto Karppanen, Matti Siekkinen, and Antti Ylä-Jääski, "Practical power modeling of data transmission over 802.11g for wireless applications", Proceedings of the ACM International Conference on Energy-Efficient Computing and Networking, 2010.

[17] D. Vardalis and V. Tsaoussidis, “Energy-efficient internetworking with DTN”, Technical Report: TR-DUTH-EE-2011-4, http://www.intersys-lab.org/pages/publications.php

[18] A. Bakre, B. R. Badrinath, "Implementation and Performance Evaluation of Indirect TCP”, IEE Transaction on Computers, Vol. 6, No. 3, 1997 